\title{
Understanding Urea Assimilation and its Effect on Lipid Production and Fatty Acid Composition of Scenedesmus Sp
}

\author{
Saumya Dhup, ${ }^{1 *}$ Dheeban C. Kannan ${ }^{2}$ and Vibha Dhawan ${ }^{2}$ \\ ${ }^{1}$ Centre for Bioresources and Biotechnology, TERI University, 10, Institutional Area, Vasant Kunj, New Delhi 110070, India \\ ${ }^{2}$ Biotechnology and Management of Bioresources Division, the Energy and Resources Institute (TERI), India Habitat \\ Centre, Lodhi Road, New Delhi 110003, India
}

Received: 9 May, 2016; Accepted: 24 May, 2016; Published: 28 May, 2016

*Corresponding author: Saumya Dhup, Centre for Bio resources and Biotechnology, TERI University, 10, Institutional Area, Vasant Kunj, New Delhi 110070, India, Tel : +91-8860262363; E-mail: saumya.dhup.18@gmail.com

\begin{abstract}
Much advancement has been made in reducing the cost of largescale production and harvesting of bio fuels. Nutrients contribute to one of the major cost components in algal production; hence, it is essential to understand the importance of minimizing costs at the nutrient level. In the current study, evaluation of urea as a lowcost and efficient source of nitrogen was investigated. The effect of its uptake mechanisms on growth, change in lipid productivity and fatty acid composition of Scenedesmus sp. was explored. Total Disappearance Rate (TDR) and total uptake rate were used to study the efficiency of urea as a nitrogen source. It was found that the nutrient uptake efficiency of urea was higher than that of nitrate. In addition, urea showed an increase in biomass productivity and lipid productivity by $26 \%$ and $45 \%$, respectively, when compared to control. Results also demonstrated degradation of urea into ammonia upon uptake by algal cells, which is then diffused out of the cells into the medium. Ammonia present in the medium is converted into ammonium ion with simultaneous degradation into nitrate. Eventually, both ammonia and nitrate are absorbed by cells in the final growth phase. These results suggest that urea can be effectively used as an alternative nitrogen source.
\end{abstract}

Keywords: Scenedesmus sp; Urea uptake mechanism; Nutrient uptake efficiency; Lipid productivity; Fatty acid composition

\section{Introduction}

Microalgae are known for their remarkable potential as a feedstock for biofuels and proteins due to their environmentfriendly conversion and enhanced yields. However, the challenges faced while using microalgae at every step of production add to the cost of the end F product. Thus, realizing the importance of overcoming these challenges, constant efforts are being made to develop efficient methods for reducing the cost. High lipid productivity of dominant, fast-growing algae is a major prerequisite for the commercial production of microalgal biodiesel.

Biomass generation by photosynthetic microalgae varies depending on a number of environmental factors, including nitrogen concentration, temperature and light intensity. Nitrogen is one of the essential nutrients critical for the cultivation of algae due to its role in growth and regulation of metabolism [1]. It is well documented that there are three different sources of nitrogen such as nitrate, ammonia and urea, which are absorbed by microalgae $[2,3]$. Urea is known to contain approximately $46.7 \%$ nitrogen content, that is, $0.467 \mathrm{Kg}$ of urea- $\mathrm{N}$ and hence it is considered as a low-cost and efficient form of nitrogen when compared to other sources $[4,5]$. Under typical water conditions, urea gives rise to nutrients such as $\mathrm{N}, \mathrm{P}$ and $\mathrm{C}(100 \%)$ and thus is supplied as a chemical fertilizer to the culture medium for algal uptake [4]. Conversely, nitrate (sodium or potassium salt) provides only $16 \%$ of nitrogen, $100 \%$ of which is readily available for the culture due to its efficient water-soluble property [5]. The above-mentioned increase in the availability of nitrogen source through urea as compared to other sources of nitrogen is observed due to the presence of two nitrogen atoms per mole of urea. Urea also proves to be an efficient alternative source to other nitrate forms as its cost of production and price per $\mathrm{kg}$ of $\mathrm{N}$ available is much lower (price per kg of sodium nitrate is 3.1 times that of urea; Table 1).

The mechanism of uptake of each nitrogen source varies, eventually changing its pathway for metabolism. Previous studies suggest that the nitrate and ammonium sources have the simplest uptake mechanisms, as they are taken up as nitrate and ammonium ion, respectively, from the medium under sufficient sunlight during photosynthesis; however, much less is known about urea uptake [6]. In addition, urea, as a nitrogen

Table 1: Comparison of cost per kg of urea and sodium nitrate with respect to availability of nitrogen $(\mathrm{N})^{23,24}$.

\begin{tabular}{|l|l|l|l|}
\hline & $\begin{array}{l}\text { Cost (Rs/ } \\
\text { kg) }\end{array}$ & $\begin{array}{l}\text { N available } \\
\text { (\%) }\end{array}$ & $\begin{array}{l}\text { Cost of N available (Rs/ } \\
\text { kg) }\end{array}$ \\
\hline Urea & $5-9$ & 46.7 & $11.56-19.26$ \\
\hline $\begin{array}{l}\text { Sodium } \\
\text { nitrate }\end{array}$ & $31-37$ & 16.5 & $187.8-224.2$ \\
\hline
\end{tabular}


source, cannot be directly assimilated, thus involving a complex mechanism [7]. Suggested that carbon dioxide and ammonia released upon urea degradation are eventually absorbed by microalgae [7]. Conversion of urea, its removal and uptake from the culture have seldom been reported.

$$
\begin{array}{ll}
\mathrm{NH}_{2}-\mathrm{CO}-\mathrm{NH}_{2}+\mathrm{H}_{2} \mathrm{O} & \mathrm{CO}_{2}+2 \mathrm{NH}_{3} \\
\mathrm{NH}_{3}+\mathrm{H}_{2} \mathrm{O} & \mathrm{NH}_{4}^{+}+\mathrm{OH}^{-}
\end{array}
$$

Both ammonia-based chemical fertilizers and urea accumulate ammonia in water in an unionized form. Therefore, in order to study the effect of an alternative source of nitrogen such as urea on the algal cell growth and understand the mechanism of conversion of urea into other nitrogen sources and their uptake, experiments were performed in a semi-continuous culture system of Scenedesmus sp. The effect of urea on growth, lipid productivity and fatty acid composition were studied to evaluate its efficacy as a major nitrogen source.

\section{Materials and Methods}

\section{Microalgal strain and culture conditions}

The freshwater microalgae Scenedesmus sp. (TX3) was procured from Hauz Khas lake park, Hauz Khas, India. The culture was maintained in Bold's basal medium (BBM) which consisted of the following components (g/l): $\mathrm{KH}_{2} \mathrm{PO}_{4}$ (0.175) $\mathrm{CaCl}_{2} \cdot 2 \mathrm{H}_{2} \mathrm{O}(0.025), \mathrm{MgSO}_{4}(0.075), \mathrm{K}_{2} \mathrm{HPO}_{4}(0.075), \mathrm{NaCl}(0.025)$, $\mathrm{H}_{3} \mathrm{BO}_{3}(0.115), \mathrm{NaNO}_{3}(0.25), \mathrm{Na}_{2}$ EDTA (0.001), $\mathrm{KOH}(6.2), \mathrm{FeSO}_{4}$ (4.98) and conc. $\mathrm{H}_{2} \mathrm{SO}_{4}(1 \mathrm{ml})$ [8]. The $\mathrm{pH}$ of the medium was adjusted to 6.8 . The medium was sterilized at $121^{\circ} \mathrm{C}$ for $15 \mathrm{~min}$ The axenic cultures were maintained in 250-ml glass jars as stock and incubated at $25 \pm 2{ }^{\circ} \mathrm{C}$.

\section{Experimental design}

Experiments were designed with four biological replicates in two similar batches - experimental and control in a semicontinuous mode. The factors differentiating the batches were based on alternate sources of nitrogen such as urea in experimental batch and nitrate in control batch. Duplicates for each biological replicate were taken for measurement at every step. Sodium nitrate has already been reported to be a typical nitrogen source for BBM; therefore, it was considered as control [8]. A pre-incubation step was carried out in which sodium nitrate was replaced with urea for acclimatization of the strain in medium with nutrient urea. This culture was then used as an inoculum and added into a 1-L Erlenmeyer flask containing a working volume of $800 \mathrm{ml}$. An initial inoculum of the strain was added at a proportion of $10 \%(\mathrm{v} / \mathrm{v})$ into the solution. As shown in Figure 1, the experiment was conducted for three passages. The results of the third passage were recorded for analysis. The inoculum used for the third phase was $50 \%(\mathrm{v} / \mathrm{v})$ of the previous passage. Therefore, the culture for the final phase was inoculated at a proportion of $50 \%(\mathrm{v} / \mathrm{v})$. The culture flasks were maintained under continuous agitation at $160 \mathrm{rpm}$ under 16:8 h light-dark photoperiod conditions. Removal of different sources of nitrogen from the medium including ammonium, nitrate and urea was evaluated. The obtained data were analysed statistically using one-way analysis of variance (ANOVA).

\section{Analytical method}

Growth Measurements: Culture growth was observed by measuring the optical density at $680 \mathrm{~nm}$ using a Shimadzu spectrophotometer. Sampling ( $3 \mathrm{ml}$ ) was done every 4 days for growth kinetics and in the late exponential phase for lipid and fatty acid methyl ester (FAME) analyses. The dry cell weight was determined by filtering $3 \mathrm{ml}$ of the cell culture through a 0.22 -mm-pore-size glass fibre filter (oleic acid). The filters with the biomass were washed with water and dried in a hot air oven at $100^{\circ} \mathrm{C}$. The increase in weight of the dry filter was measured.

Nutrient Analysis: Culture samples $(3 \mathrm{ml})$ were taken at an interval of 4 days until they were harvested for nutrient measurements. The samples collected were filtered through glass fibre filters for analysis. Nutrients were analysed photo metrically using the standard methods developed by the American Public Health Association (APHA). The total disappearance rate (TDR) was calculated by summation of the disappearance rates of all nitrogen forms in a particular medium. The nutrient disappearance rate (NDR, mg-Nutrient/L/day) was calculated as follows:

$\mathrm{NDR}=\left(C_{\mathrm{o}}-C_{\mathrm{t}}\right) / t$

Where $C_{\mathrm{o}}$ and $C_{\mathrm{t}}$ are the nutrient concentrations $(\mathrm{mg} / \mathrm{l})$ at the beginning and end of the experiment, respectively, and $t$ is the time interval (days).

The percentage of stored nitrogen reserves within an algal cell was calculated using a CHN elemental analyser (Thermo Finnigan, San Jose, CA, USA). The nutrient uptake rate (NUR, mgNutrient/L/day) refers to the amount of nutrient taken up by unit mass of algae and it was calculated on the basis of dry cell weight as follows:

NUR $=\left(D_{\mathrm{f}} \times N_{\mathrm{f}}-D_{\mathrm{i}} \times N_{\mathrm{i}}\right) / t$

Where $D_{\mathrm{f}}$ and $D_{\mathrm{i}}$ are the final and initial dry cell weight in $\mathrm{mg} / \mathrm{l}$, respectively, and $N_{\mathrm{i}}$ and $N_{\mathrm{f}}$ denote the percentage of the

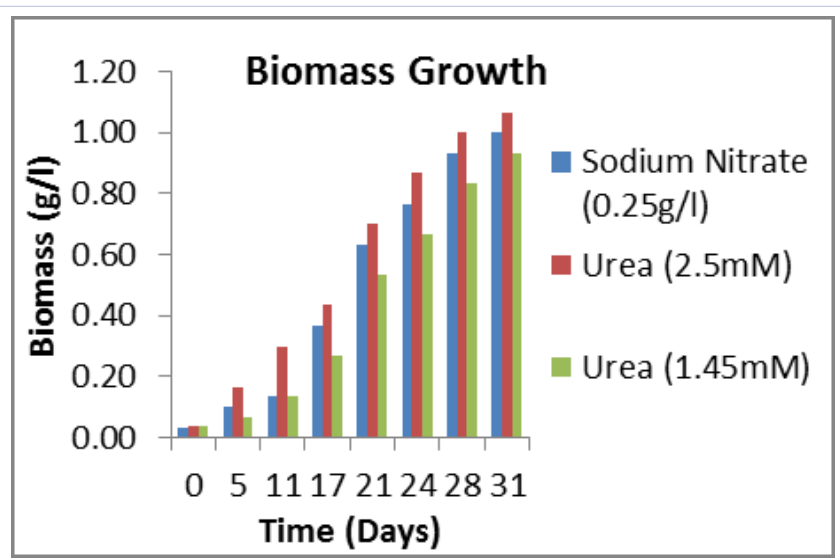

Figure 1: Optimization of two different concentrations of urea, graph represents the biomass growth for sodium nitrate and urea at two different concentrations. 


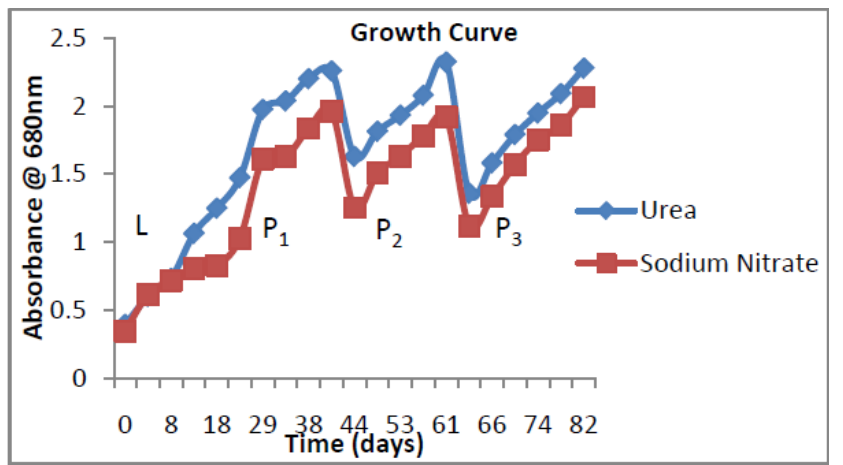

Figure 2: Periodic fluctuation of growth in semi-continuous culture: L represents pre-culture period; P1, P2 and P3 are nutrient recharge and biomass removal intervals.

initial and final concentration of nitrogen, respectively, present in the algal biomass.

\section{Lipid extraction, esterification and fatty acid analysis}

The two batches of samples were harvested by continuous centrifugation (8000 rpm for $5 \mathrm{~min}$ ). The concentrated algal samples were frozen overnight at $-80^{\circ} \mathrm{C}$ and freeze-dried under vacuum. The algal mass was accurately weighed, and lipid extraction was carried out according to the method adopted by [9].

Lipid content (\%) and lipid productivity (g/L/day) were calculated as follows:

Lipid content $\left(C_{\text {lipid }}\right)=($ wt. of lipid $/$ wt. of sample $) \times 100$

Lipid productivity $=\left(C_{\text {lipid }} \times \mathrm{DCW}\right) / \mathrm{t}$

Where, $C_{\text {lipid }}$ is the lipid content (\%), DCW the dry cell weight ( $\mathrm{g} / \mathrm{l}$ ) and $t$ the time interval (days).

Lipid extracts were converted to methyl esters with methanolic $\mathrm{HCl}$ and hexane. FAMEs were prepared by adding 1 $\mathrm{ml}$ of concentrated $\mathrm{HCl}$ along with $5 \mathrm{ml}$ methanol to the methyl esters. The mixture was heated at $80-90^{\circ} \mathrm{C}$ in a water bath for 30 min. Then, $1 \mathrm{ml}$ of hexane was added to the vial after methylation. The top hexane layer containing the methyl esters was placed into gas chromatography (GC) vials for subsequent GC analysis (Agilent 6890N, Agilent Technologies, Palo Alto, CA, USA) using a DB-5 column $(0.2 \mathrm{~mm}$ ID, $30 \mathrm{~m}, 0.25 \mathrm{~mm}$ film thickness; Agilent Technologies, Palo Alto, CA, USA) equipped with a flame ionization detector. The temperature programme consisted of an initial temperature of $2^{\circ} \mathrm{C}$ and followed by an increase of $50^{\circ} \mathrm{C} \mathrm{min}{ }^{-1}$ up to $250^{\circ} \mathrm{C}$. The peaks were integrated using the Chemstation and identified by comparing the retention times with the pure standard (Sigma). The system's performance was checked with blanks and standard samples prior to analysis. Concentrations were expressed in $\mathrm{mg} / \mathrm{ml}$ and then converted to percentage. All the tests were performed in triplicates.

\section{Results and Discussion}

\section{Effect of urea on growth kinetics}

$\mathrm{BBM}$, best known for culturing green algae, provides an optimal environment for a large variety of microalgal species. It is essential to understand that each algal species has specific culture conditions, and thus its optimization is very crucial. As nutrients are one of the major cost components in algal production, they have to be optimized efficiently for a higher productivity. An optimal nutrient condition is attributed to the combined effect of both concentration and source of nutrient [10]. Conducted a study on S. bijugata and reported the significance of selecting a suitable nitrogen source and concentration for rapid growth [10]. The authors also stated that the association between the source of nitrogen and concentration is significant. In this study, an alternative source of nitrogen was considered because nitrogen, being one of the major basic elements, often limits growth and accumulation of lipids. The effect of urea on growth and lipid productivity of Scenedesmus sp. was investigated. The study was carried out to evaluate the use of a cost-efficient alternative of nitrogen in the medium. The amount of urea and sodium nitrate added in the medium is indicated in Table 2 . The concentration of urea in the medium was optimized previously for growth and lipid productivity. In an earlier study, two different molar concentrations of urea were tested: $1.45 \mathrm{mM}$ (equiatomic to sodium nitrate) and $2.5 \mathrm{mM}$ (equivalent weight basis: $17.6 \mathrm{mM}$ ). As the urea concentration $(2.5 \mathrm{mM})$ in the medium was higher than the equiatomic concentration of sodium nitrate, it resulted in higher biomass growth (Figure 1) and lipid productivity (12.4 $\mathrm{mg} / \mathrm{l} /$ day). The lipid productivity with urea concentration of $1.45 \mathrm{mM}$ and sodium nitrate was $7.3 \mathrm{mg} / \mathrm{l} /$ day and $8.8 \mathrm{mg} / \mathrm{l} /$ day respectively. It can be inferred that an increased concentration of urea provides an increased amount of nitrogen which leads to higher growth and productivity [10]. Observed that an optimal higher concentration of urea results in better biomass growth [10]. Therefore, the concentration of nitrogen available after conversion of urea is observed to be optimum, thereby enhancing lipid productivity. Urea is also known to enhance growth rate as it acts as a complementary source of organic carbon. Thus, the combined effect of nitrogen and carbon simultaneously leads to an increase in the growth of Scenedesmus sp.

The concentration of the nutrient in the medium varies according to the nutrient source used. A change in the nutrient source might lead to an increased concentration in the medium above the tolerance level of the algal cells; such high concentrations are toxic and inhibit cell growth [11]. But an appropriate amount of nitrogen concentration is known to increase the microalgal growth rate [12]. In this study, it was observed that the difference in the available forms of the nitrogen

Table 2: Growth parameters and productivity of Scenedesmus sp.

\begin{tabular}{|l|l|l|}
\hline N Source & Urea & Sodium Nitrate \\
\hline Amount (mM) & 2.5 & 2.9 \\
\hline Biomass productivity (g/l/day) & 0.048 & 0.038 \\
\hline Specific growth rate (day $\left.\mathbf{- 1}^{-1}\right)$ & 0.027 & 0.018 \\
\hline Lipid content (\%) & $8.7 \pm 0.4$ & $7.6 \pm 0.6$ \\
\hline Lipid productivity (mg/l/day) & 4.176 & 2.88 \\
\hline
\end{tabular}




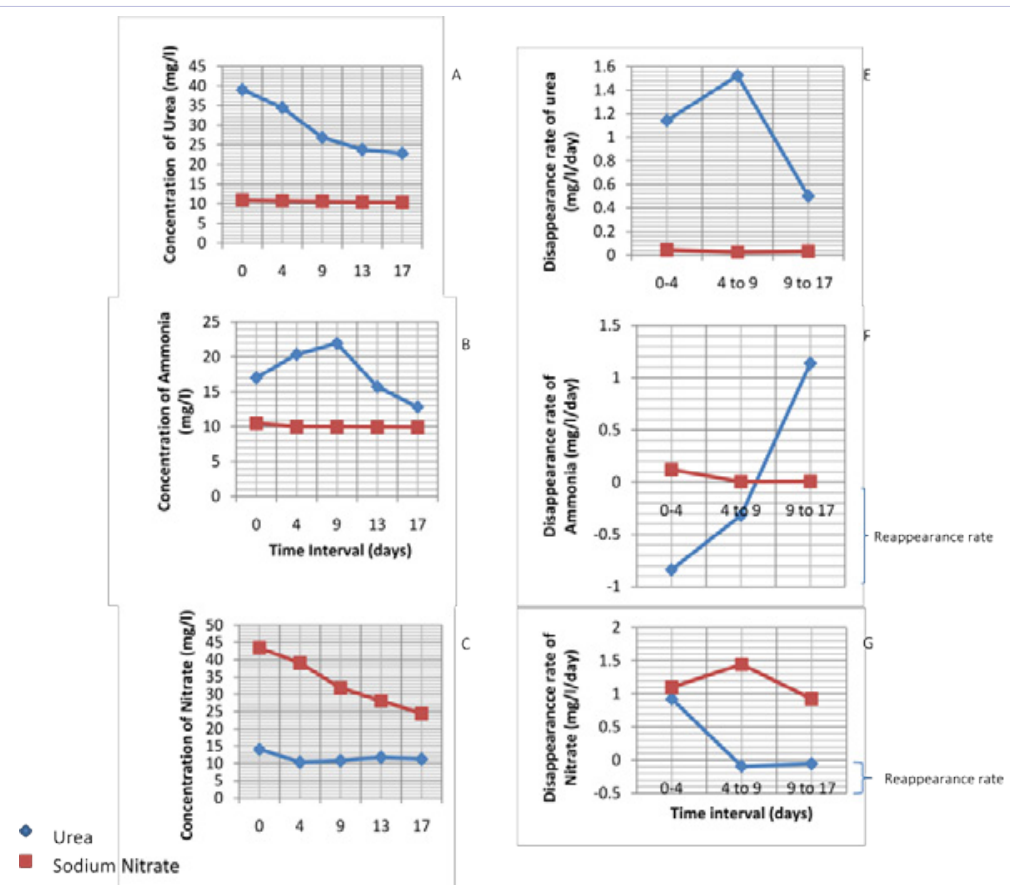

Figure 3: Disappearance of nitrogen forms from the medium containing urea (experimental) versus sodium nitrate (control).

Figures A, B and C show concentrations of urea, ammonium and nitrate at disappearance, respectively

$\mathrm{E}, \mathrm{F}$ and $\mathrm{G}$ show the disappearance rate of urea, ammonium and nitrate, respectively.

for intake leads to a change in the nutrient concentration in the medium and thus causes variations in the assimilation rate. These alterations subsequently affect the growth rate of the algal species.

Growth curves for Scenedesmus sp. grown in two nitrogen sources are presented in Figure 2. In the medium containing urea, a higher exponential increase in the growth curve is observed with a specific growth rate of 0.06 day $^{-1}$. However, in the medium with sodium nitrate, specific growth rate was $0.05 \mathrm{day}^{-1}$. Therefore, it is evident from Table 2 that the specific growth rate of this species is significantly affected by the nitrogen source used $(P<0.05)$, as the growth rate with urea in the medium is 1.4 times higher than with nitrate. These results suggest that urea is more preferred as a nitrogen source in the medium [13]. Reported a similar growth pattern in the Chlorella sp. with urea and sodium nitrate as the two nitrogen sources and their specific growth rates being 0.071 day $^{-1}$ and 0.047 day $^{-1}$, respectively $[10,13]$. In their study on Scenedesmus sp. also reported that the amount of biomass obtained with urea in the medium was equivalent to that with nitrate [10]. Previous reports on phytoplankton species suggest that urea is degraded into ammonia inside the cells [13]. In this study, ammonia, a product of urea degradation in the medium with urea, is being preferred over nitrate in the batch having sodium nitrate as the nitrogen source [14]. Observed a similar result wherein some microalgal species prefer ammonium ion over nitrate $[14,15]$. Reported that Scendesmus sp. grew faster in the stable phase of growth when the ammonium form of nitrogen was provided in the medium [15]. They stated that in accordance with the specific growth rate, the preference of nitrogen source is in the order of ammonium < urea < nitrate. Ammonia is considered as a preferred source for intake by the cells because it is directly absorbed into the cell and converted into amino acids which are eventually used for metabolic activities. When nitrate as a nitrogen source is absorbed by the cells, it reaches the cytosol where it gets reduced to nitrite by nitrate reductase. Nitrite produced is transported immediately to the chloroplast where it is further reduced to ammonium ion by nitrite reductase [16]. Therefore, the algal cells prefer a source which is directly assimilated and used for metabolic activities. The results suggest that ammonium ion is a much preferred nitrogen source for Scenedesmus sp. Therefore, the production of ammonium ions during urea degradation makes this nutrient more suitable for algal growth than sodium nitrate.

The above-mentioned results show a variation in the growth characteristics based on two different nitrogen sources, and a change in the biomass concentration is also expected. As shown in Table 2, a difference between the biomass productivity of urea and sodium nitrate was observed. The medium with urea showed an increase in biomass productivity by $26 \%$ in 17 days with a biomass concentration of $0.832 \mathrm{~g} / \mathrm{l}$. Similar results with an enhanced biomass dry weight were reported with urea in Scenedesmus sp [17]. They reported that Scendesmus sp. performed better in a medium with urea, resulting in a significantly higher biomass concentration and lipid productivity when compared to nitrate. It was also observed that the percentage of nitrogen (calculated using an elemental analyser; Thermo Finnigan, San Jose, CA, USA) inside the cells was higher in the medium with urea $(8.179 \%$ in medium with urea and $7.219 \%$ 
Table 3: Nutrient disappearance and uptake efficiency for Scenedesmus sp. in medium with urea and sodium nitrate.

\begin{tabular}{|c|c|c|c|c|c|c|c|c|c|}
\hline \multirow[b]{2}{*}{ Days } & \multicolumn{3}{|c|}{$\begin{array}{l}\text { Total Disappearance Rate } \\
\text { (mg/l/day) }\end{array}$} & \multicolumn{3}{|c|}{ Total Uptake Rate (mg/l/day) } & \multirow{2}{*}{$\begin{array}{l}\text { Total Disappearance } \\
\text { Rate } \\
0-17 \\
\end{array}$} & \multirow{2}{*}{$\begin{array}{l}\text { Nutrient } \\
\text { Uptake Rate }\end{array}$} & \multirow{2}{*}{$\begin{array}{l}\text { Nutrient } \\
\text { Uptake } \\
\text { Efficiency (\%) }\end{array}$} \\
\hline & $0-4$ & $4-9$ & $9-17$ & $0-4$ & $4-9$ & $9-17$ & & & \\
\hline Urea & 1.22 & 1.11 & 1.58 & 1.11 & 1.1 & 1.54 & 1.3636 & 1.3117 & $96.00 \%$ \\
\hline Sodium Nitrate & 1.26 & 1.47 & 0.96 & 1.12 & 1.45 & 0.9 & 1.1845 & 1.1182 & $94.00 \%$ \\
\hline
\end{tabular}

with nitrate). This suggests that using urea as the nutrient source leads to a higher nitrogen uptake efficiency, thereby increasing the amount of nitrogen in vivo and also resulting higher biomass concentration.

\section{Uptake mechanism of urea and sodium nitrate}

This study focuses on urea as an alternative nitrogen source and its impact on growth, lipid production and fatty acid composition. The impact is observed largely due to the difference in the uptake mechanisms of the nitrogen sources such as urea and sodium nitrate. Studies related to the uptake mechanism of urea by algal species are limited. In 1988, Price and Harrison conducted a study proposing a model of urea uptake and its assimilation by a diatom, Thalassiosira pseudonana in nitratesufficient and nitrate-starved cells [13]. They have explained the diffusion of urea through an ammonia conversion route using radioactive isotopes. They proposed that upon cellular uptake, urea gets degraded into ammonia which is released. The ammonia reacts with water and is converted into ammonium ion, thereby releasing hydroxide ion in the medium. This mechanism can be further explored in other species in order to assess and confirm the consumption of urea by other phytoplankton communities. In this study, a similar trend was observed using different analytical methods for uptake and assimilation. It has been previously studied that ammonia-based chemical fertilizers such as urea accumulate a unionized form of ammonia in the medium. The unionized form of ammonia is taken up biologically by the algal culture during photosynthesis in sufficient light intensity before it gets oxidized to nitrate, thus maintaining the culture alkalinity [18]. But it was also observed that a small quantity of ammonia which was diffused out from the cells was converted into ammonium ion and nitrate simultaneously [18]. The nitrate and ammonium ion were eventually reabsorbed by the cells. Thus, based on this study, it can be assumed that under light and dark conditions both nitrogen sources, ammonium ion and nitrate, respectively, are simultaneously available in a medium with urea.

The rate of urea disappearance from the medium after 4 days averaged $1.14 \mathrm{mg} / \mathrm{l} /$ day. But the maximum disappearance rate of urea from the medium was recorded as $1.52 \mathrm{mg} / \mathrm{l} /$ day for an interval of 4-9 days. With the disappearance of urea, there was an exponential increase in the reappearance of ammonium ion in the medium (Figure. 3E and F). This suggests that urea absorbed by the cells was degraded in vivo into ammonia. Ammonia was then diffused out into the medium. Degradation of urea inside the cell was carried out in the presence of urease enzyme present in most algal cells [19]. The ammonia diffused out was eventually taken up as ammonium ion, as during the final interval of cell growth, the rate of disappearance of ammonium ion from the medium was observed to be $1.13 \mathrm{mg} / \mathrm{l} /$ day (Figure 3F). While ammonia disappeared during the final phase, simultaneous reappearance of nitrate in the medium was observed at a rate of $0.1 \mathrm{mg} / \mathrm{l} /$ day (Figure 3G).

When considering the rate of uptake, the overall disappearance of nitrogen source from the medium was taken into account over the period. The uptake rate in the medium with urea for an interval of 0-4 days was observed to be more than that for a period of 4-9 days $(1.11 \mathrm{mg} / \mathrm{l} /$ day and $1.10 \mathrm{mg} / \mathrm{l} /$ day, respectively). The TDR was also observed to be higher for the interval of 0-4 days (Table 3). In the final phase of growth, the maximum disappearance rate was observed due to the simultaneous uptake of both ammonium ion and nitrate nitrogen from the medium with urea. Thus, an increase in the rate of uptake of the nitrogen source was observed. In the medium with nitrate, the maximum disappearance and uptake rates was observed for an interval from 4 to 9 days. It was also observed that the nutrient uptake efficiency of medium with urea was $96 \%$, while that of medium with sodium nitrate was $94 \%$ (Table 3 ). Thus, the results indicated an enhanced uptake of nutrient from the medium with urea in comparison to the medium with sodium nitrate. It can be concluded that urea is a better nitrogen source as it is being efficiently absorbed and utilized for metabolic functions leading to enhanced growth rate and lipid productivity.

\section{Effect of urea on lipid productivity and fatty acid composition}

Biomass productivity is considered as an imperative parameter for determining the effect of a condition. Lipid content is another parameter which is essential for the evaluation of the overall performance of the strain. In the current study, the lipid content and biomass were considered as the twin effects for evaluating the overall lipid productivity under different nutrient sources.

We proceeded to analyse the total lipid content of the strain in both media (experimental and control) by the end of the cycle where minimal nutrients were left in the medium. Harvesting in the late exponential phase leads to a decrease in the nitrogen and phosphorous concentrations but an increase in the lipid content [12]. Table 2 shows the total lipid content and productivity of the two media. It was observed that the total lipid content of the batch with urea $(8.7 \%)$ was higher than that of sodium nitrate 
Table 4: Fatty acid profile of Scenedesmus sp. in medium with urea and sodium nitrate.

\begin{tabular}{|l|l|l|}
\hline & Urea (\%) & Sodium nitrate (\%) \\
\hline C14 & 0.36 & 0.76 \\
\hline C14:1 & 0.13 & 0.39 \\
\hline C15 & 0.41 & 0.44 \\
\hline C15:1 & 1.86 & 2.78 \\
\hline C16 & 5.40 & 9.00 \\
\hline C16:1 & 3.49 & 17.70 \\
\hline C17 & 0.73 & 0.75 \\
\hline C17:1 & 1.58 & 2.51 \\
\hline C18 & 0.00 & 0.00 \\
\hline C18:1 & 14.16 & 8.99 \\
\hline C18:2 TRANS & 1.05 & 5.44 \\
\hline C18:3 & 61.04 & 37.84 \\
\hline C18:3 (6) & 2.55 & 4.13 \\
\hline C18:3 (3) & 2.79 & 3.97 \\
\hline C20 & 2.60 & 0.96 \\
\hline C22:2 & 0.58 & 2.03 \\
\hline C20:2 & 0.60 & 0.80 \\
\hline C21:1 & 0.52 & 0.71 \\
\hline C24:1 & 0.16 & 0.40 \\
\hline SFA & 9.50 & 11.90 \\
\hline MUFA & 21.90 & 33.47 \\
\hline PUFA & 68.60 & 54.23 \\
\hline
\end{tabular}

(7.6). Due to a $14 \%$ increase in the lipid content of the strain in the medium with urea, a difference between the lipid productivity of the two media was observed. The lipid productivity in the batch with urea was found to be 1.5 times higher than that with sodium nitrate. It was inferred that the lipid content increases with an increase in biomass productivity. Hence, the lipid productivity of the cells in the batch with urea increased. Similar results were also indicated in previous studies carried out by [20]. Thus, the results listed in Table 2 shows that the lipid content and productivity were significantly dependent on the nitrogen source provided $(P>0.05)$. These results indicate that nitrogen in the medium with urea should be provided in sufficient amount for the cells to grow and store lipids efficiently. More importantly, in the medium with urea, the presence of ammonia makes it possible for the cells to absorb it as a direct nitrogen source for assimilation. Direct assimilation of ammonia facilitates rapid cell metabolism, thereby increasing the lipid productivity.

Fatty acid composition is another key parameter that determines the potential of an algal strain as a biodiesel candidate. In this particular study, fatty acid analysis of the species in the two different media gave interesting insights with a prominent shift in the fatty acid composition. The major saturated, monosaturated and polyunsaturated fatty acids were found to be C16:0, C16:1 and $\mathrm{C} 18: 1$ and C18:3, respectively. According to the previous studies conducted on Scenedesmus sp., stearic acid was absent in both media [21]. As shown in Table 4, there is a significant visible shift of the fatty acid percentage from C16:0 and C16:1 to C18:1 and C18:3 in the medium with urea; the percentage of the latter increased by 5 and 23, respectively [22] (Table 4). Observed a similar shift from palmitic acid to oleic acid in other feedstock soybean, but stated that such an increase in oleic acid was not due to environment influences [22]. But, herein, the shift to oleic acid with a significant increase in the level of an essential omega-3 fatty acid (alpha-linolenic acid) was observed due to the change in nitrogen source. An increase in oleic acid makes the strain desirable as a potential source for biodiesel, as monounsaturated fatty acids are most suitable when considering cloud point and oxidative stability. In addition, an increased amount of saturated fatty acids poses problems with gelling of fuel in cold weather.

Due to the presence of a high amount of omega-3 fatty acids, this strain is an important source of essential nutrients and also presents health benefits apart from biodiesel production; however, it negatively influences the oxidative stability. Therefore, the percentage of essential fatty acids of the total algal lipids in urea was $94 \%$ (significant percentages of $\alpha$-linolenic acid and oleic acid were $61 \%$ and $14 \%$, respectively), whereas in the medium with sodium nitrate, it was $74 \%$. Hence, it can be concluded that with a change in the nitrogen source from nitrate to urea, there is an increase in lipid productivity with a positive shift in the fatty acid composition from non-essential to essential fatty acids.

\section{Conclusion}

The growth and lipid productivity of green algae Scenedesmus sp. was tested in BBM medium containing a low-cost nitrogen source. Our findings shed light on the use of urea as a nitrogen source, as it proved to be more efficient in terms of growth, lipid productivity and fatty acid composition when compared to sodium nitrate. With regard to the nutrient uptake mechanism and fate of urea in Scenedesmus sp., it was observed that the results are in compliance with previous studies conducted on other phytoplankton species, with a reasonably higher nutrient uptake efficiency than nitrate. Although sodium nitrate is a readily available source of nitrate ion, the ammonium ion obtained after urea degradation is a much-preferred source of nitrogen for uptake. In this study, the presence of nitrate as an additional nitrogen source along with ammonium ion was observed. The nitrogen obtained upon degradation of ammonia was also observed to be absorbed by the cells. Hence, urea can be effectively used as an alternative economical substitute of nitrogen for growth and lipid production of Scenedesmus sp. at a large scale.

\section{Highlights}

- Urea is considered as a low-cost alternative source of nitrogen.

- A higher uptake efficiency of urea (two nitrogen sources including ammonium ion and nitrate) is observed when compared to sodium nitrate. 
- A change in the fatty acid composition is observed upon urea uptake.

\section{Acknowledgement}

We sincerely thank the Department of Biotechnology, Government of India for funding the research. We also thank the Director General, TERI, for providing the necessary infrastructural services and facilities for this study as well as Late Dr. Prem Dureja, Ms V Devi and Ms Swati Patel for their valuable guidance during the research.

\section{References}

1. Xian-Ming Shi, Feng Chen, Jian-Ping Yuan, Hui Chen. Heterotrophic production of lutein by selected Chlorella strains. J Appl Phycol 1997;9(5): 445-50.

2. Ludwig, C. A. The availability of different forms of nitrogen to a green alga. Am J Bot. 1938;25(6):448-58.

3. Prabakaran P, Ravindram AD. Influence of different carbon and nitrogen sources on growth and $\mathrm{CO} 2$ fixation of microalgae. Adv Appl Sci Res. 2012;3 (3): 1714-17.

4. Knud-Hansen, Batterson CF TR, CD, McNabb S. Harahat, K Sumantadinata and H.M. Eidman . Nitrogen input, primary productivity and fish yield in fertilized freshwater ponds in Indonesia. Aquacul. 1991;94: 49-63.

5. Harben PW, Theune C. Nitrogen and nitrates. In: Kogel JE, Trivedi NC, Barker JM. and Krukowski ST. (eds.) Industrial Minerals \& Rocks: Commodities, Markets, and Uses, Littleton, CO, Society for Mining, Metallurgy and Exploration, Inc. 2006;671-78.

6. Kim SY, Lee, Hwang SJ. Removal of nitrogen and phosphorous by Chlorella sorokiniana cultured heterotrophically in ammonia and nitrate. Intl Biodeterio Biodegrad. 2013;85:511-16.

7. Price NM, Harrison PJ. Uptake of urea $\mathrm{C}$ and urea $\mathrm{N}$ by the coastal marine diatom Thalassiosira pseudonana. Limnol oceanogr. 1988;33(4): 528-37.

8. Bischoff HW, Bold HC. Phycological studies IV. Some soil algae from enchanted rock and related algal species. University of Texas Publication. 1963;6318: 1-95.

9. Folch J, Lees M, Sloane Stanley GH. A simple method for the isolation and purification of total lipids from animal tissues. J Biol Chem. 1957;226(1):497-509.

10. Arumugam M, Agarwal A, Arya MC, Ahmed Z. Influence of nitrogen sources on biomass productivity of microalgae Scenedesmus sp. Bioresour Technol. 2013;131:246-9. doi: 10.1016/j. biortech.2012.12.159.

11.Xu D, Gao Z, Zhang X, Qi Z, Meng C, Zhuang Z. Evaluation of the potential role of the macroalga Laminaria japonica for alleviating coastal eutrophication. Bioresour Technol. 2011;102(21):9912-8. doi: 10.1016/j.biortech.2011.08.035

12. DhupS, Dhawan V. Effect of nitrogen concentration on lipid productivity and fatty acid composition of Monoraphidium sp. Bioresour Technol. 2014;152:572-5. doi: 10.1016/j.biortech.2013.11.068.

13. El-Sayed, AB, Abel-Maguide AA. Growth response of Chlorella vulgaris to acetate carbon and nitrogen forms. Nature Sci. 2011;9(9).53.

14. Kang YH, Park SR, Chung IK. Bio filtration efficiency and biochemical composition of three seaweed species cultivated in a fish-seaweed integrated culture. Algae. 2011;26(1):97-108. DOI: 10.4490/ algae.2011.26.1.097.

15. Xin L, Hong-ying H, Ke G, Jia Y. Growth and nutrient removal properties of a freshwater microalga Scendesmus sp. LX1 under different kinds of nitrogen sources. Ecol Eng. 2010;36(4): 379-81.

16. Sakihama Y, Nakamura S, Yamasaki H. Nitric oxide production mediated by nitrate reductase in the green alga Chlamydomonas reinhartii; An alternate no production pathway in photosynthetic organisms. Plant Cell Physiol. 2002;43(3):290-7.

17. Ying Shen, Zhijian Pei, Wenqiao Yuan, Enrong Mao. Effect of nitrogen and extraction method on algae lipid yield. Intl J Agricul Biol Eng. 2009;2(1): 51-57. DOI: 10.3965/j.issn.1934-6344.2009.01.051-057

18. Sugiyama M, Kawai A. Microbiological studies on the nitrogen cycle in aquatic environments-VI. Metabolic rate of ammonium nitrogen in a goldfish culturing pond. B Jap Soc Sci Fish. 1979;45(6):785-789. DOI: 10.2331/suisan.45.785.

19. Bekheet IA, Kandil KM, Shaban NZ. Studies on urease extracted from Ulva lactuca. Hydrobiol. 1984;116(1): 580-83

20. Ren HY, Liu BF, Ma C, Zhao L, Ren NQ. A new lipid- rich microalga Scenedesmus sp. strain R-16 isolated using Nile red staining: effects of carbon and nitrogen sources and initial $\mathrm{pH}$ on the biomass and lipid production. Biotechnol Biofuels. 2013;6(1):143. doi: 10.1186/17546834-6-143.

21. Choi KJ, Nakhost Z, Krukonis VJ, Karel M. Supercritical fluid extraction and characterization of lipids from algae Scenedesmus obliquus, Food Biotechnol. 1987;1(2):263-81.

22.Graef G, LaVallee BJ, Tenopir P, Tat M, Schweiger B, Kinney AJ, et al. A high-oleic-acid and low-palmitic-acid soybean: agronomic performance and evaluation as a feedstock for biofuel. Plant Biotechnol J. 2009;7(5):411-21. doi: 10.1111/j.1467-7652.2009.00408.x.

23. Market price. http://www.needsinfo.com/globalinquirymagazine/ market_price.htm

24. Chemical prices remain steady. Buisness standards. http://www. business-standard.com/article/markets/chemical-prices-remainsteady-111020800160_1.html. 